\title{
Aerobic Bacteriological Profile of Surgical Site Infection and their Antimicrobial Resistance Pattern at a Tertiary Care Hospital
}

\author{
A. Venkata Raghavendra Rao*, Reena Rajan and R. Indra Priyadharsini
}

Department of Microbiology, VMKV Medical College and Hospital, Vinayaka Mission's Research Foundation (Deemed to be university), Salem, Tamilnadu, India

\section{A B S T R A C T}

\begin{tabular}{|l|}
\hline Ke y w o r d s \\
$\begin{array}{l}\text { Surgical site } \\
\text { infection, Gram } \\
\text { negative bacilli, } E . \\
\text { coli, Multidrug } \\
\text { resistant Klebsiella } \\
\text { spp, MRSA }\end{array}$ \\
\hline Article Info \\
\hline $\begin{array}{l}\text { Accepted: } \\
\text { 04 June } 2019 \\
\text { Available Online: } \\
\text { 10 July } 2019\end{array}$ \\
\hline
\end{tabular}

\section{Introduction}

Surgical site infections (SSI's) are the third most common cause of hospital acquired infections with a reported incidence rate of 1416\% (Hohmann et al., 2012). In India, the overall incidence of wound sepsis ranges from 10\%-33\% (Bangal et al., 2014). The increased rates of surgical site infection are associated with high morbidity and mortality and are likely to have an important role in the development of antibiotic resistance (Reiye
Surgical site infections are most common nosocomial infection and are associated with prolonged hospital stay, health care cost, morbidity and mortality. The causative agents involved in SSI's may vary with geographical location, between surgeons, various procedures and from hospital to hospital. The present study was done to determine the aerobic bacterial flora from surgical site infections and their antimicrobial resistance pattern. A total of 50 culture positive samples obtained from SSI's were included in this study. Isolates were identified by standard biochemical methods and their antimicrobial resistance pattern was determined by Kirby Bauer Disk Diffusion method. Among the total 65 isolates obtained, Gram negative bacilli 45/65 (69.23\%) were the most common isolate. The most predominant organism isolated were E. coli $(24.62 \%)$ followed by Klebsiella spp (21.54\%), Proteus spp (12.31\%) and Pseudomonas spp (10.77\%). Among the Gram positive organism isolated, (21.54\%) were Staphylococcus aureus followed by Enterococcus spp (7.70\%) and Coagulase negative Staphylococcus (1.54\%). All isolates of Gram negative bacilli showed sensitivity to Imipenem. The most effective drug against gram positive organism was found to be Linezolid. For effective control of wound infection, knowledge of causative organisms, their antibiotic sensitivity patterns and administration of judicious therapy are necessary. 
infection in the tissues of the incision and operative area that can commonly occurs between the fifth and 30th days post-surgery.

Most SSI's are caused by the normal flora of patient's skin, mucous membrane or hollow viscera. The most frequently isolated pathogens are Staphylococcus aureus, Enterobacteriaceae, Coagulase Negative Staphylococci (CONS), Enterococci and Pseudomonas aeruginosa. The risk of infection depends on various factors such as size of inoculums, virulence and the ability of the organism to invade tissue (Bastola et al., 2017). The development of surgical site infection depends on several factors such as microbial pathogenicity, host defences, local environmental factors and surgical techniques (Mundhada and Tenpe, 2015; Khyati Jain et al., 2014; Arvind Kurhade et al., 2015). Dirty and contaminated wounds reflect the number of pathogens present at the operation site at the time of surgery. Prolonged use of drain may act as a pathway for pathogenic bacteria to enter the wound and thereby increasing the risk of infection.

Etiological agents involved in SSI's may vary with geographical location, between surgeons, various procedures, from hospital to hospital or even in different wards of the same hospital (Arvind Kurhade et al., 2015). Data regarding the causative organisms, their antibiotic sensitivity patterns and administration of judicious therapy are necessary for effective control of wound infection.

\section{Aims and Objectives}

To find the aerobic bacterial flora from surgical site infections.

To determine the antibiogram and the antimicrobial resistance pattern of isolates from wound infection.

Materials and Methods
Type of Study: Cross sectional study

Study period: 6 months (March-August 2018)

A total of 50 samples obtained from Surgical site infections (SSI's) received in the Department of Microbiology, VMKVMCH, Salem were included in this study.

\section{Inclusion criteria}

Patients of all age group.

Patients undergoing clean surgeries.

\section{Exclusion criteria}

Contaminated surgeries.

Infection occurring 30 days after operation if no implant is in place.

Infection on Episiotomy wounds.

\section{Sample collection}

Samples were taken from patient before wound cleaning with antiseptic solution. Two wound swabs were collected under aseptic precautions from deep inside wound avoiding contact with skin commensals. For provisional diagnosis, gram staining was done on smear made from one swab. The other swab was inoculated onto Blood agar and MacConkey agar and incubated overnight at $37^{\circ} \mathrm{C}$. Bacterial isolates were identified by colony morphology and biochemical characteristics (Collee, 2006).

\section{Detection of antimicrobial resistance}

Antimicrobial susceptibility testing was done on these isolates by Kirby Bauer Disk Diffusion method as per CLSI guidelines using the following antibiotics - Amoxycillin /

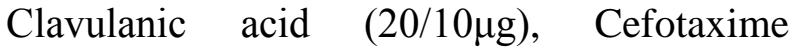


$(30 \mu \mathrm{g})$, Gentamicin $(10 \mu \mathrm{g})$, Ciprofloxacin $(10 \mu \mathrm{g})$, Cotrimoxazole $(25 \mu \mathrm{g})$, Erythromycin $(15 \mu \mathrm{g})$, Clindamycin $(2 \mu \mathrm{g})$, Linezolid $(30 \mu \mathrm{g})$, Teicoplanin $(30 \mu \mathrm{g})$, Ceftazidime $(30 \mu \mathrm{g})$, Cefepime $(30 \mu \mathrm{g})$, Imipenem $(10 \mu \mathrm{g})$ and Piperacillin / Tazobactam $(100 / 10 \mu g)$. Methicillin resistance is detected by using Cefoxitin disc $(30 \mu \mathrm{g})$ (10). Staphylococcus aureus - ATCC 25923, Escherichia coli ATCC 25922 and Pseudomonas aeruginosa ATCC 27853 were used as control strains (CLSI 2016).

\section{Results and Discussion}

Out of 50 culture positive samples studied, 40 samples showed monomicrobial growth and 10 showed polymicrobial growth (Fig. 1). A total of 65 isolates were obtained from 50 samples. Gram negative bacilli 45/65 (69.23\%) were the most common isolate obtained (Table 1).

The most predominant organism isolated were E.coli 16/65 (24.62\%) followed by Klebsiella spp 14/65 (21.54\%), Proteus spp 8/65 $(12.31 \%)$ and Pseudomonas spp $7 / 65$ $(10.77 \%)$.

Among the Gram positive organisms, 14/65 (21.54\%) were Staphylococcus aureus, followed by Enterococcus spp 5/65 (7.70\%) and Coagulase negative Staphylococcus 1/65 $(1.54 \%)$.

$30(60 \%)$ out of 50 samples were from male patients and 20 (40\%) were from female patients.

$10 / 50(20 \%)$ cases were from patients in the age group of 21-40 years.

Majority of the isolates were obtained from patients in the age group of 41-60 years $(46 \%)$.

Among the gram negative bacilli, 10/16 (63\%)
E.coli were resistant to Cephalosporins, 9/16 (56\%) were resistant to Ciprofloxacin and $8 / 16 \quad(50 \%)$ isolates were resistant to Piperacillin / Tazobactam (Fig. 2). The most effective drugs against $E$. coli were Imipenem, Cefoperazone / sulbactam and Aminoglycosides.

10/14 (71\%) Klebsiella spp were resistant to Cefoperazone / Sulbactam, 11/14 (79\%) were resistant to Cephalosporins, 8/14 (57\%) were resistant to Piperacillin / Tazobactam and 7/14 $(50 \%)$ isolates were resistant to Ciprofloxacin (Fig. 3). Imipenem was found to be the most effective antibiotic against multidrug resistant Klebsiella spp.

2/8 (25\%) isolates of Proteus spp were resistant to Cephalosporins, Amoxycillin Clavulanate and Gentamicin (Fig. 4). Isolates of Proteus showed $100 \%$ susceptibility to Imipenem and Piperacillin Tazobactam.

100\% Pseudomonas isolates showed susceptibility to Ciprofloxacin and Imepenem. 4/7 (57\%) were resistant to Cephalosporins and $3 / 7(43 \%)$ were resistant to Cefoperazone / Sulbactam (Fig. 5).

$6(42.86 \%)$ out of 14 Staphylococcus aureus isolates were resistant to Cefoxitin and were identified as MRSA. All S. aureus isolates were resistant to Penicillin. 5/14 (35.71\%) were resistant to Clindamycin, 7/14 (50\%) to Erythromycin and 13/14 (92.86\%) to Ciprofloxacin. All isolates were sensitive to Linezolid (Table 2).

Four (80\%) out of 5 isolates of Enterococcus were resistant to Penicillin, Erythromycin and Ciprofloxacin and 1/5 (20\%) isolates were resistant to Linezolid. A single isolate of CONS obtained from SSI's was found to be sensitive to Erythromycin, Clindamycin, Cefoxitin and Linezolid. 
Fig.1 Aerobic bacterial growth pattern from surgical site infections

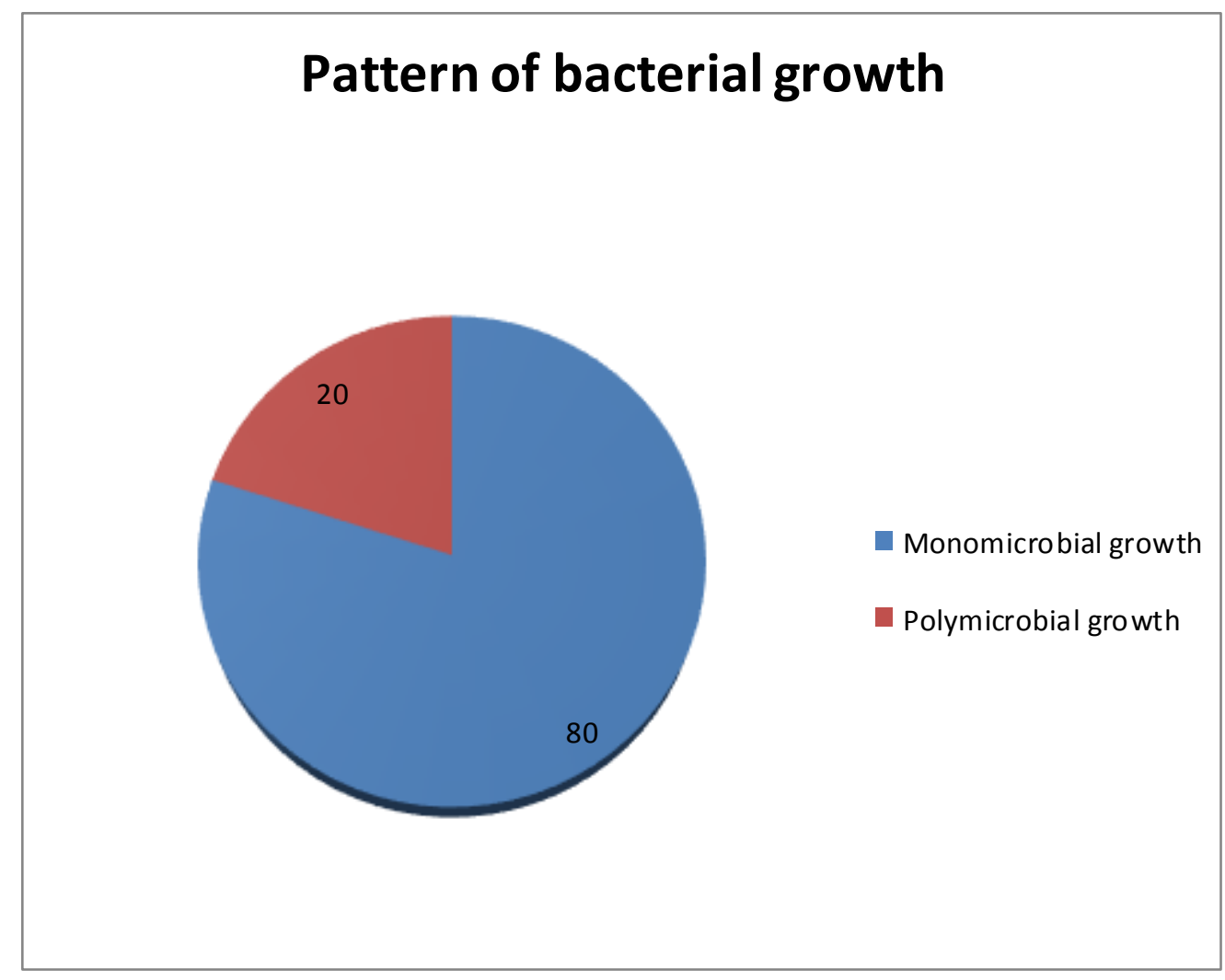

Fig.2 Antimicrobial resistance pattern of E. coli

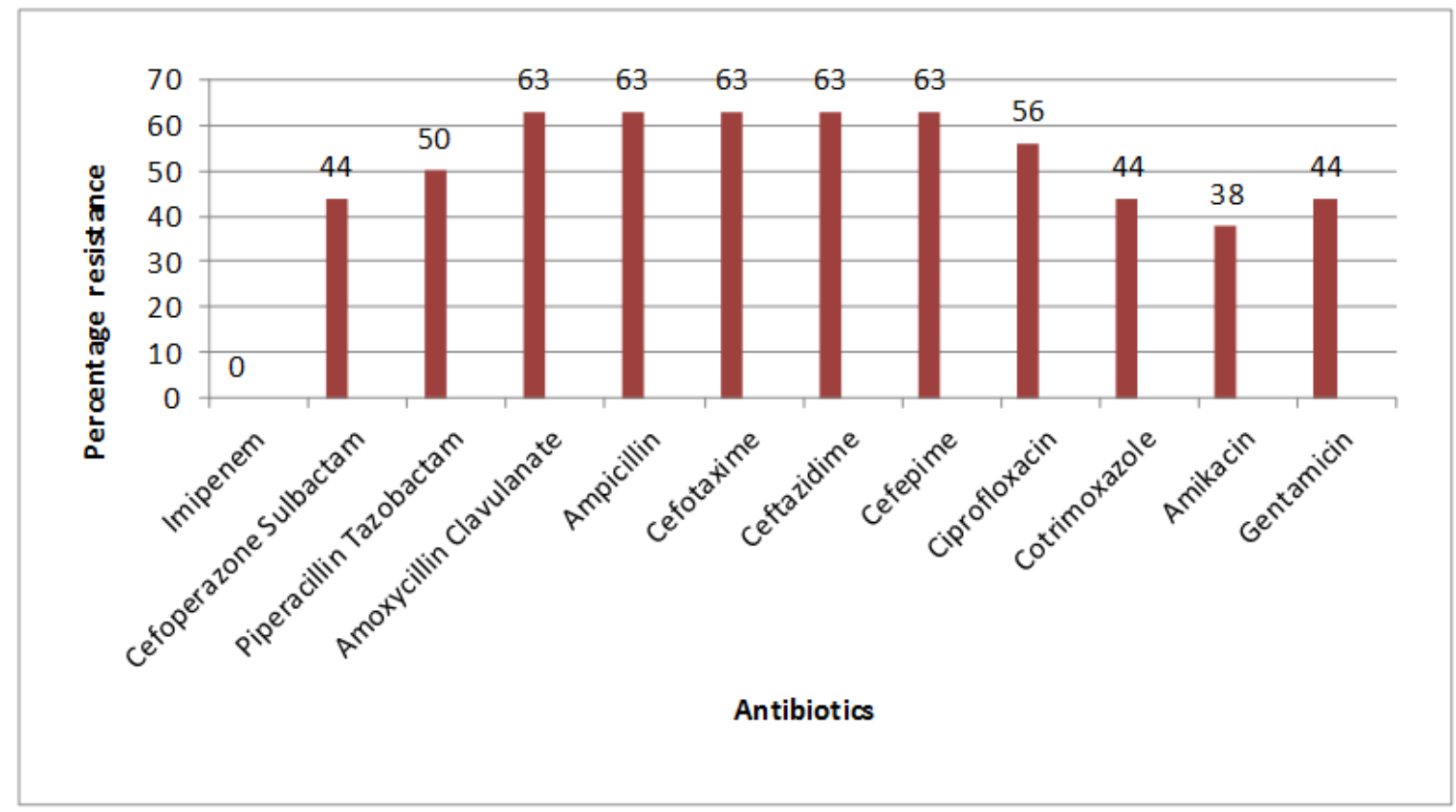


Fig.3 Antimicrobial resistance pattern of Klebsiella spp

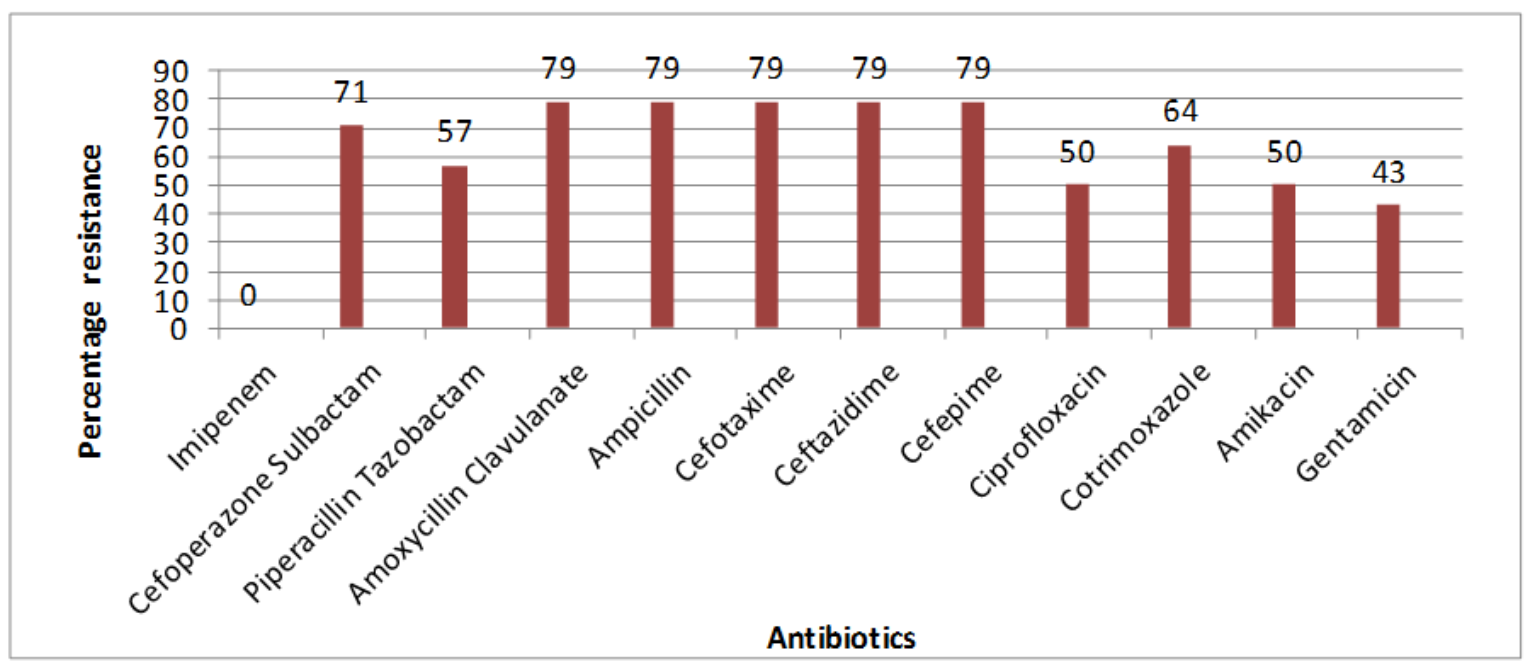

Fig.4 Antimicrobial resistance pattern of Proteus spp

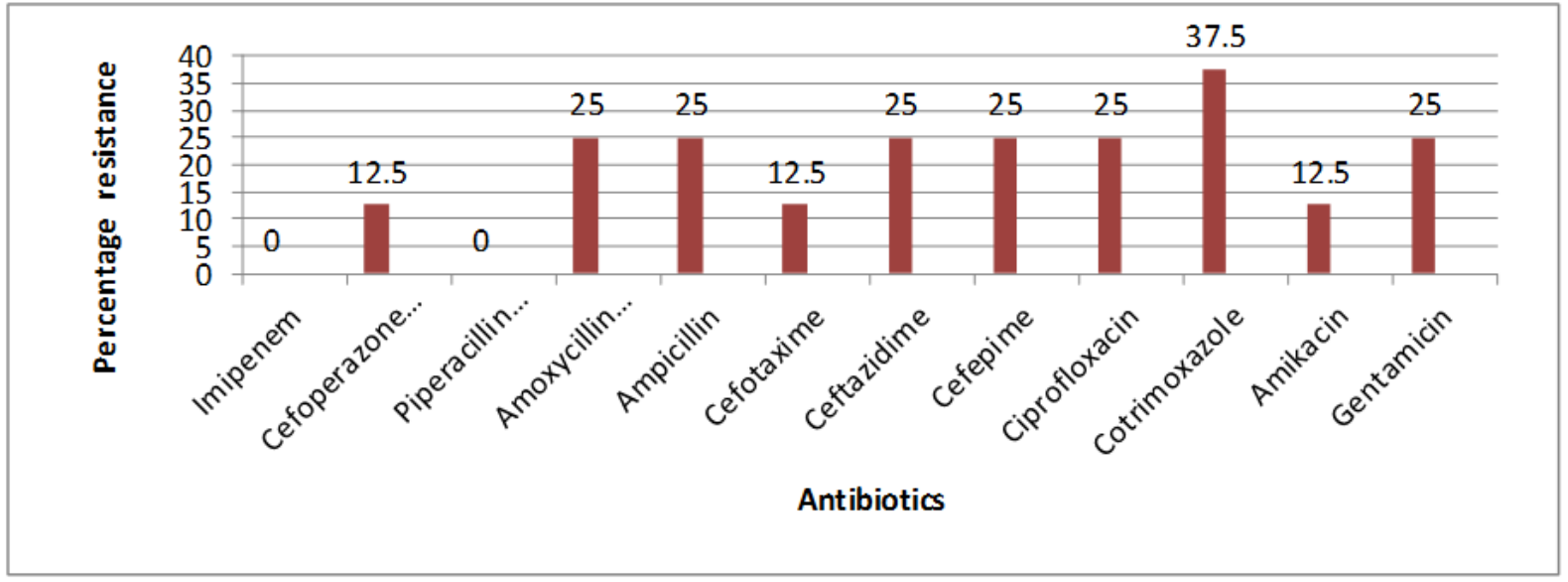

Fig.5 Antimicrobial resistance pattern of Pseudomonas spp

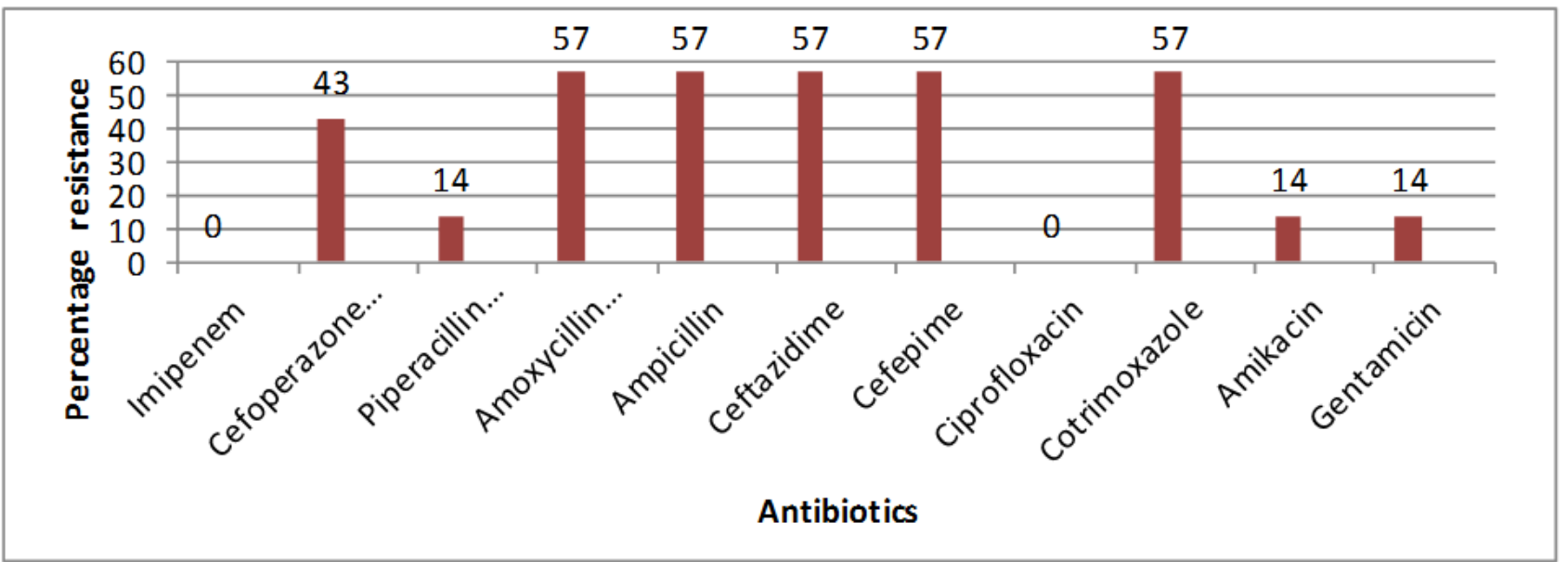


Table.1 Distribution of Polymicrobial growth

\begin{tabular}{|l|l|}
\hline Ward & Organisms \\
\hline Surgery Unit I & $\begin{array}{l}\text { E.coli \& CONS } \\
\text { Klebsiella \& Proteus }\end{array}$ \\
\hline MICU & $\begin{array}{l}\text { E.coli \& Enterococcus } \\
\text { Klebsiella, Pseudomonas \& Proteus }\end{array}$ \\
\hline Surgery Unit II & $\begin{array}{l}\text { E.coli \& Proteus } \text { (2 cases) } \\
\text { Pseudomonas \& MRSA } \\
\text { MRSA \& Enterococci }\end{array}$ \\
\hline Surgery Unit III & Klebsiella \& Pseudomonas \\
\hline
\end{tabular}

Table.2 Antimicrobial resistance pattern of Gram positive cocci

\begin{tabular}{|c|c|c|c|c|c|c|c|c|}
\hline Organism & \multicolumn{7}{|c|}{ Antibiotics (\%) } \\
\hline & CIP & CO & AK & G & E & CD & P & LZ \\
\hline MSSA (8) & $8(100)$ & $8(100)$ & $3(38)$ & $5(63)$ & $3(38)$ & $3(38)$ & $8(100)$ & 0 \\
\hline MRSA (6) & $5(83)$ & $4(67)$ & $3(50)$ & $3(50)$ & $4(67)$ & $2(33)$ & $6(100)$ & 0 \\
\hline Enterococcus (5) & $4(80)$ & $4(80)$ & $1(20)$ & $4(80)$ & $4(80)$ & $4(80)$ & $4(80)$ & $1(20)$ \\
\hline CONS (1) & $1(100)$ & $1(100)$ & $1(100)$ & $0(0)$ & $0(0)$ & $0(0)$ & $1(100)$ & $0(0)$ \\
\hline
\end{tabular}

CIP- Ciprofloxacin; CO- Cotrimoxazole; AK- Amikacin; G- Gentamicin; E- Erythromycin; CD- Clindamycin; PPenicillin; LZ- Linezolid

Despite the introduction of meticulous antiseptic regime in surgical practice, SSI is the most common surgical complication in developed and developing countries and is associated with prolonged hospital stay, increased hospital readmissions, health care costs and increased morbidity and mortality.

Prolonged preoperative hospital stay leads to colonization with antimicrobial resistant pathogens. These organism directly affect patient's susceptibility to infection either by lowering host resistance or by providing increased opportunity for ultimate bacterial colonization.

The present study showed predominance of Gram negative bacterial isolates from surgical site infections. Various studies from India have reported the predominance of Gram positive cocci from post-operative wound infection. In a study report by Goswami et al., 2011, $68.85 \%$ isolates were gram negative organisms from SSI's. In our study, 80\% samples from SSI s showed monomicrobial growth and $20 \%$ were Polymicrobial. A study conducted by Khyati Jain et al., 2014 has reported $92.30 \%$ isolates as monomicrobial and $7.6 \%$ as polymicrobial from SSI's.

E.coli was the most common isolate obtained in our study; whereas a similar study by Shinde and Kulkarni 2017 have reported S.aureus as common organism from SSI's, followed by E.coli, Citrobacter, Pseudomonas and Acinetobacter. Kokate et al., (2017) have reported $29.31 \%$ Pseudomonas aeruginosa from SSI's followed by $25.86 \% \mathrm{E}$. coli. The high incidence of gram negative bacilli in the postoperative wound infection may be attributed to be acquired from normal endogenous flora of patients. Among the Gram positive isolates obtained, S.aureus was the common organism. S.aureus is most likely associated 
as normal flora of skin and anterior nares and also with contamination from environment, surgical instrument and from hands of health care workers.

In our study, majority of the isolates were obtained from male patients in the age group of 41-60 years. This is similar to the study findings by Naveen et al., (2014), who have reported the increase in frequency of surgical site infection with age. This may be due to poor immune response, existing comorbidities in aged patients and reduced compliance with treatment. In another study, Bastola et al., (2017) have shown increased isolation rate from male patients. Pooja patel et al., 2019 have reported maximum number of cases from patients in the age group of 4858 years. Vikrant Negi et al., (2015) have reported the incidence of SSI among patients above 50 years with $E$. coli as common organism isolated.

All isolates of Gram negative bacilli were sensitive to Imipenem in our study. Least susceptibility was observed against Cephalosporins and Ciprofloxacin. Among the Enterobacteriaceae, Klebsiella spp was found to be more resistant to antimicrobial agents compared to E. coli and Proteus. More than $70 \%$ isolates of Klebsiella were resistant to Cephalosporins and Cefoperazone sulbactam. Isolates of Klebsiella showed 100 $\%$ susceptibility to Imipenem. The most effective antibiotic against $E$. coli was Imipenem and Cefoperazone sulbactam.

More than $50 \%$ isolates of E.coli were resistant to fluoroquinolone and Cephalosporins. In a similar study conducted by Pooja Patel et al., (2019), 100 \% E. coli isolates were sensitive to Imipenem. In a study report by Preethishree et al., (2017), $100 \%$ E. coli isolates showed susceptibility to Imipenem and Piperacillin Tazobactam. In our study, isolates of Pseudomonas aeruginosa showed $100 \%$ sensitivity to Imipenem and Ciprofloxacin. Kurhade et al., (2015) have reported $48.5 \%$ isolates of Pseudomonas resistant to Ciprofloxacin.

The prevalence of MRSA from SSI's was found to be $42.86 \%$. Bhave PP et al., 2016 have reported $8.6 \%$ MRSA from postoperative wound infection. In another study report Kokate et al., (2017) have showed 25\% MRSA isolates from SSI's and 39.7 \% MRSA were reported by Sudhaharan et al., (2018).

All isolates of staphylococcus aureus were resistant to penicillin.100 \% isolates were found to be susceptible to Linezolid. This is similar to the study findings by Mundhada and Tenpe (2015).

Among the Gram positive isolates studied, drug resistance was found to be higher among Enterococcus isolates. $80 \%$ isolates were resistant to Penicillin, Erythromycin, Aminoglycosides and Ciprofloxacin and 20\% isolates were resistant to Linezolid. Isolates of Enterococci exhibit inherent resistance to antimicrobial agents such as Penicillins, Cephalosporins, Aminoglycosides and Clindamycin.

These organisms also acquire resistance via mobility of resistance genes on plasmids, transposons and chromosomal exchange (Seema Sood et al., 2008).

The present study showed predominance of multidrug resistant Klebsiella spp from surgical site infection. SSI's contribute significantly to the cost, the morbidity, and the possible long-term consequences of a surgical procedure. Surveillance of SSI with feedback of appropriate data to surgeons would be desirable to reduce the rate of infection and formulate a proper antibiotic policy for reducing the abuse of antibiotics thereby preventing antimicrobial resistance. 


\section{References}

Aniruddha S. Mundhada, Sunita Tenpe. A study of organisms causing surgical site infections and their antimicrobial susceptibility in a tertiary care Government Hospital. Indian Journal of Pathology and Microbiology, 2015; 58:195-200.

Arvind Kurhade, Suresh Akulwar, Meena Mishra, Geeta Kurhade, Angel JustizVaillant, Krutika Kurhade, Sehlule Vuma and Sudhir Lakhdive. Bacteriological Study of Post-Operative Wound Infections in a Tertiary Care Hospital. Journal of Bacteriology and Parasitology, 2015; 6(6): 1-4

Bangal, V.B., Borawake SK, Shinde KK, Gavhane SP. Study of surgical site infections following gynaecological surgery at tertiary care teaching hospital in Rural India. International Journal of Biomedical Research, 2014; 05(02): 113-16.

Bastola R, Parajuli P, Neupane A, Paudel A. Surgical Site infections: Distribution Studies of Sample, Outcome and Antimicrobial Susceptibility Testing. Journal of Medical Microbiology and Diagnosis, 2017; 6: 252

Bhave PP, Kartikeyan S, Ramteerthakar MN, Patil NR. Bacteriological study of surgical site infections in a tertiary care hospital at Miraj, Maharashtra state, India. International Journal of Research in Medical Sciences, 2016; 4: 26302635.

Clinical and Laboratory Standard Institute (CLSI). Performance standards for antimicrobial susceptibility testing. 26th ed. Wayne USA.CLSI, 2016.

Collee JG, Marr W. Specimen collection, culture containers and media. In: Collee JG, Marmion BP, Fraser AG, Simmons A, editors. Mackie and McCartney Practical Medical Microbiology. 14th ed. London, Churchill Livingstone; 2006; p. 95-111.

Goswami NN, Trivedi HR, Goswami AP, Patel TK, Tripathi CB. Antibiotic sensitivity profi le of bacterial pathogens in postoperative wound infections at a tertiary care hospital in Gujarat, India. Journal of Pharmacology and Pharmacotherapeutics, 2011; 2(3):158-64.

Hohmann C, Eickhoff C, Radziwill R, Schulz M. Adherence to guidelines for antibiotic prophylaxis in surgery patients in German hospitals: a multicentre evaluation involving pharmacy interns. Infection, 2012; 40(2): 131-37.

Khyati Jain, Nilesh Shyam Chavan S.M. Jain. Bacteriological profile of post-surgical wound infection along with special reference to MRSA in central India, Indore. International Journal of Integrative Medical Sciences, 2014; 1(1): 9-13.

Patel Pooja, Patel HK, Nerurkar AB. Antimicrobial susceptibility pattern of organisms causing surgical site infection in a tertiary care hospital, Valsad, South Gujarat. Indian Journal of Microbiology Research, 2019; 6(1):7177.

Preethishree P., Rekha Rai and K. Vimal Kumar. Aerobic Bacterial Profile of Post-Operative Wound Infections and their Antibiotic Susceptibility Pattern. International Journal of Current Microbiology and Applied Science. 2017; 6(9): 396-411.

Reiye Esayas Mengesha, Berhe Gebre-Slassie Kasa, Muthupandian Saravanan, Derbew Fikadu Berheand Araya Gebreyesus Wasihun. Aerobic bacteria in post-surgical wound infections and pattern of their antimicrobial susceptibility in Ayder Teaching and 
Referral Hospital, Mekelle, Ethiopia BMC Research Notes, 2014; 7:575

Sandeep Bhaskarrao Kokate, Vaishali Rahangdale, Vyankatesh Jagannath Katkar. Study of bacteriological profile of post-operative wound infections in surgical wards in a tertiary care hospital. International Journal of Contemporary Medical Research, 2017; 4(1):232-235.

Seema Sood, Meenakshi Malhotra, B K Das, Arti Kapil. Enterococcal infections and antimicrobial resistance. Indian Journal of Medical Research, 2008; 12(2):111121.

Setty Naveen H, Nagaraja MS, Nagappa DH, Giriyaiah CS, Gowda NR, Laxmipathy Naik RD. A study on Surgical Site Infections (SSI) and associated factors in a government tertiary care teaching hospital in Mysore, Karnataka. International Journal of Medicine and Public Health, 2014; 4:171-175
Shinde A, Kulkarni S. Study of Organisms causing Surgical Site Infections and their Antimicrobial Susceptibility Pattern in Rural Teaching Hospital. MIMER Medical Journal, 2017; 1(2):912.

Sukanya Sudhaharan, Padmaja Kanne, Padmasri Chavali, Lakshmi Vemu. Aerobic bacteriological profile and antimicrobial susceptibility pattern of pus isolates from tertiary care hospital in India. The Journal of Infection in Developing Countries, 2018; 12(10):842-848.

Vikrant Negi, Shekhar Pal, Deepak Juyal, Munesh Kumar Sharma, Neelam Sharma. Bacteriological Profile of Surgical Site Infections and Their Antibiogram: A Study From Resource Constrained Rural Setting of Uttarakhand State, India. Journal of Clinical and Diagnostic Research. 2015; 9(10):17-20.

\section{How to cite this article:}

Venkata Raghavendra Rao, A., Reena Rajan and Indra Priyadharsini, R. 2019. Aerobic Bacteriological Profile of Surgical Site Infection and their Antimicrobial Resistance Pattern at a Tertiary Care Hospital. Int.J.Curr.Microbiol.App.Sci. 8(07): 113-121. doi: https://doi.org/10.20546/ijcmas.2019.807.015 